\title{
Politische Voraussetzungen von Austeritätspolitik: Ein internationaler Vergleich von 17 etablierten Demokratien zwischen 1978 und 2009
}

von Klaus Armingeon, Kai Guthmann und David Weisstanner

Welche politischen Voraussetzungen müssen gegeben sein, damit Regierungen den Staatshaushalt konsolidieren? In diesem Beitrag konzentrieren wir uns auf zwei Erklärungsgrößen: Die parteipolitische Zusammensetzung einer Regierung und die Breite der Regierungskoalition. Wir zeigen einerseits, dass die Wahrscheinlichkeit der Initiierung eines Konsolidierungsprogramms unter Parteien der politischen Rechten sowie unter kleinen Reformkoalitionen (Einparteienregierungen und minimal winning Koalitionen) am höchsten ist. Andererseits finden wir ein nahezu entgegengesetztes Muster bezüglich des Umfangs der verabschiedeten Sparmaßnahmen. Hier zeigt sich, dass die Breite der Regierungskoalition grundsätzlich einen positiven Einfluss auf die Größe des Konsolidierungsprogramms ausübt. Zudem fallen die Sparpakete rechter Parteien generell bescheidener aus als jene ihrer Konkurrenten der politischen Mitte und Linken. Wir erklären diese Ergebnisse mit den strategischen und wahlpolitischen Optionen der Akteure, auf Basis eines Vergleichs von 17 OECD-Ländern zwischen 1978 und 2009.

Under what political conditions do governments consolidate their public finances? In this article we focus on two factors: the partisan complexion of governments and the breadth of the governing coalition. We demonstrate that the probability for a government to initiate a fiscal consolidation program is highest under parties of the political right, as well as, under narrow reform coalitions (which we operationalize as one party governments and minimal winning coalitions). We observe the exact opposite when it comes to the extent of these adjustment measures. Here, the breadth of the policy coalition exerts a positive influence. Along these lines, consolidation packages implemented by right parties are smaller in size than those of their centrist or leftist competitors. On the basis of a quantitative analysis of 17 OECD countries between 1978 and 2009, we explain these findings with the strategic and electoral options of political actors.

\section{Einleitung}

Auch wenn Regierungsrhetoriken suggerieren, alle gesellschaftlichen Gruppen würden von Programmen zur fiskalischen Konsolidierung gleichmäßig getroffen, 
sind die empirischen Befunde eindeutig: Die Kosten zahlen vor allem jene im unteren Teil der Einkommenshierarchie. ${ }^{1}$ Unterstellt man rationales Verhalten, sollten sich diese Gruppen - und die mit ihnen historisch oder aufgrund des Wahlverhaltens besonders verbundenen Parteien der politischen Linken - dagegen wehren. Hingegen sollten liberale und konservative Regierungsparteien weniger Skrupel haben, das Skalpell an Ausgabenposten zugunsten der Einkommensschwachen anzusetzen. Theorien der Vetopunkte lassen vermuten, dass ihnen dies besonders gut gelingt, wenn sie sich nicht mit anderen Akteuren abstimmen müssen. ${ }^{2}$ Dazu gehören in erster Linie Koalitionspartner. Deshalb würde man umfangreiche Konsolidierungsprogramme besonders dann erwarten, wenn liberale oder konservative Parteien Einparteienregierungen stellen.

Aber trifft dies auch wirklich zu? Welche politischen Voraussetzungen müssen gegeben sein, damit Regierungen den Staatshaushalt konsolidieren? Dies ist unsere Leitfrage.

Wir behaupten, dass die These der generellen Vorteilhaftigkeit von liberalen und konservativen Einparteienregierungen für die Umsetzung umfangreicher Konsolidierungsmaßnahmen zu kurz greift. Um ein vollständiges Bild der relativen Parteien- und Koalitionseffekte zu erhalten, muss zunächst zwischen der grundsätzlichen Entscheidung zur Konsolidierung einerseits und dem Umfang des Sparprogramms andererseits differenziert werden.

Wenn es darum geht, sich grundsätzlich zur Austerität zu entschließen, sollte die traditionelle Logik der Parteiendifferenzhypothese und Vetospielertheorie tatsächlich eine erhebliche Erklärungskraft aufweisen. Unter rechten Regierungen sind fiskalische Konsolidierungen demnach sowohl aus ideologischen Gründen als auch als Signal an die eigene Wählerschaft eher zu erwarten, als unter ihren Gegenspielern der politischen Mitte und Linken. Auch sollte die Anzahl der Vetospieler eine gewichtige Rolle spielen. Gerade im politisch sensiblen Bereich von üblicherweise redistributiv wirkenden Sparmaßnahmen ist die Wahrscheinlichkeit des Scheiterns zwischenparteilicher Verhandlungen umso größer, je mehr Akteure daran beteiligt sind. Unter minimalen Koalitionen (Einparteienregierungen und minimal winning Koalitionen) sollten Konsolidierungsmaßnahmen daher prinzipiell häufiger zu beobachten sein, als unter breiter angelegten

1 Armingeon, K./Guthmann, K./Weisstanner, D.: Choosing the Path of Austerity: How Policy Coalitions Shape Welfare-Policy Choices in Periods of Fiscal Consolidations, unpubliziertes Manuskript (2014); Ball, L./Furceri, D./Leigh, D./Loungani, P.: The Distributional Effects of Fiscal Consolidation, in: IMF Working Paper, WP/13/151 (2013).

2 Tsebelis, G.: Veto Players: How Political Institutions Work, Princeton, 2002. 
Bündnissen.

In der Frage nach dem Umfang eines Sparprogramms, nachdem sich alle beteiligten Akteure bereits grundsätzlich zur Austerität entschlossen haben, erwarten wir hingegen ein anderes Muster. So sollten von rechten Regierungen umgesetzte Konsolidierungen im Durchschnitt kleiner ausfallen, als unter Regierungen anderer politischer Couleur. Dies ist zum einen auf die schärfere Opposition der Wohlfahrtsstaatsklientel zurückzuführen, die rechte Regierungen zu fürchten haben, wenn sie die Kosten der Konsolidierung auf diese Gruppen zu externalisieren versuchen. Zum anderen profitieren linke und zentristische Parteien - als historische Begründer und Garanten des Wohlfahrtsstaates - von ihrer höheren Glaubwürdigkeit, auch umfassende Austeritätsprogramme sozial- und wohlfahrtsstaatsverträglich umsetzen zu können. ${ }^{3}$

Ebenso sollten Sparprogramme unter breiten Koalitionen nun, da die Grundsatzentscheidung bereits getroffen ist, größer ausfallen als unter minimalen Koalitionen. Letztere sind besonders anfällig für die politisch-elektoralen Konsequenzen von Austerität und haben einen stärkeren Anreiz, die mit Konsolidierungsmaßnahmen verbundenen Kosten zu externalisieren. Dies wiederum erhöht das Niveau gesellschaftlicher Konflikte und damit die Wahrscheinlichkeit des Zurückstutzens ambitionierter Reformpläne. In breiten Koalitionen ist eine Externalisierung der Kosten aufgrund der Einbindung wichtiger Vetospieler und der somit eher konsens-orientierten Politik nur begrenzt möglich. Zudem ist es hier weniger wahrscheinlich, dass die Kooperationsbereitschaft einiger Akteure durch oppositionelle Kräfte ausgebeutet wird. ${ }^{4}$

Wir mobilisieren empirische Evidenz für unser Argument in einer vergleichenden Untersuchung von Austeritätspolitiken in 17 entwickelten OECDDemokratien zwischen 1978 und 2009. Dabei stützen wir uns auf einen neuen Datensatz, der den Umfang fiskalischer Konsolidierungen auf Basis der von den Reformmaßnahmen zu erwartenden Einsparungen in Prozent des Bruttoinlandsproduktes beziffert.

3 Kitschelt, H.: Partisan Competition and Welfare State Retrenchment. When Do Politicians Choose Unpopular Policies?, in: Pierson, P. (Hg.): The New Politics of the Welfare State, Oxford, 2001, 265302 .

4 Jacobs, A.M.: Governing for the Long Term. Democracy and the Politics of Investment, Cambridge, 2011. 


\section{Theoretisches Argument}

Programme zur Konsolidierung des Staatshaushaltes sind in Demokratien politisch höchst anspruchsvoll. Es handelt sich dabei in der Regel um redistributive Politik. Häufig beanspruchen Politikerinnen und Politiker, die notwendigen Sparmaßnahmen sollten fair und unter besonderem Schutz der sozial Schwachen durchgeführt werden. Die Realität sieht dagegen meist anders aus. Systematisch vergleichende Untersuchungen kommen zu dem Schluss, dass Austeritätspolitiken zur Umverteilung zuungunsten der sozial Schwachen führen. ${ }^{5}$

Vergleichende sozialwissenschaftliche Analysen von redistributiver Politik bestätigen häufig die Thesen der Machtressourcen sowie der Parteiendifferenz. ${ }^{6}$ Ihnen zufolge verteidigen Gewerkschaften, linke und christdemokratische (zentristische $^{7}$ ) Parteien die Interessen der unteren Einkommensschichten an der Aufrechterhaltung oder auch Ausweitung staatlicher Umverteilung, während Unternehmer und ihre Verbände, liberale und konservative (rechte) Parteien solche Ziele in der Regel nicht unterstützen.

Man könnte deshalb erwarten, dass Austeritätspolitiken besonders stark von rechten Parteien angestrebt und in starkem Maße umgesetzt werden. Dies sollte ihnen besonders leicht gelingen, wenn es wenige Vetospieler gibt. Vetospieler sind jene Akteure, deren Zustimmung notwendig ist, um eine bestimmte Reform durchzuführen. Dazu gehören auch parteipolitische Vetospieler: Die Parteien einer Regierungskoalition. Je mehr solcher Vetospieler es gibt, desto schwieriger ist es, den Status Quo zu verändern. ${ }^{8}$ Deshalb sollte die Wahrscheinlichkeit für eine umfassende Austeritätspolitik unter rechten Einparteienregierungen am höchsten sein.

Freilich findet die empirische Literatur diesen Zusammenhang zwischen parteipolitischer Färbung und Austerität (vor allem in Bezug auf deren Auftretens-

5 Siehe z.B. Armingeon, K./Guthmann, K./Weisstanner, D.: Choosing the Path of Austerity: How Policy Coalitions Shape Welfare-Policy Choices in Periods of Fiscal Consolidations, a.a.O.; Ball, L./Furceri, D./Leigh, D./Loungani, P.: The Distributional Effects of Fiscal Consolidation, a.a.O.; Mulas-Granados, $C .:$ Fiscal Adjustments and the Short-Term Trade-Off between Economic Growth and Equality, in: Hacienda Pública Espanola / Revista de Economia Pública, 172/1 (2005), 61-92.

6 Schmidt, M.G./Ostheim, T./Siegel, N.A./Zohlnhöfer, R. (Hg.): Der Wohlfahrtsstaat. Eine Einführung in den historischen und internationalen Vergleich, Wiesbaden, 2007.

7 Unter dem Begriff der zentristischen Parteien werden hier christdemokratische oder katholische Parteien gefasst sowie einige weitere Parteien, die sich dem sozialen Ausgleich und der sozialen Sicherheit verpflichtet fühlen, wie dies beispielsweise in den katholischen Sozialenzykliken seit Rerum Novarum (1891) entwickelt wurde. Zur operationalen Definition von zentristischen Parteien siehe Armingeon, K./Knöpfel, L./Weisstanner, D./Engler, S./Potolidis, P./Gerber, M.: Comparative Political Data Set I 1960-2011, Bern, 2013.

8 Tsebelis, G.: Veto Players: How Political Institutions Work, a.a.O. 
wahrscheinlichkeit und Umfang) nur bedingt. Sparpolitiken erscheinen weitgehend als Funktion des fiskalischen Problemdrucks. Die Rolle von Parteien ist höchst bescheiden ${ }^{9}$ und zeigt sich nur vereinzelt in konditionalen und indirekten Effekten der parteipolitischen Färbung der Regierung, etwa in Abhängigkeit vom Wahlzyklus, der Knappheit der bevorstehenden Wahlen oder dem gewerkschaftlichen Organisationsgrad. ${ }^{10}$

Freilich könnte dieser fehlende Zusammenhang mit der Komposition von Sparpolitiken und den daraus resultierenden unterschiedlichen Verteilungswirkungen zu tun haben, die durchaus in Abhängigkeit der ideologischen Orientierung der Regierung variieren können. Beispielsweise würde man von linken Parteien erwarten, dass sie Haushaltsdefizite bevorzugt einnahmeseitig über höhere Steuern auf Spitzeneinkommen bekämpfen, während konservative und liberale Parteien stärker aufgabenbasiert zulasten des Wohlfahrtsstaates und damit auch der unteren Einkommenskategorien konsolidieren. In diesem Beitrag steht die Zusammensetzung von Austerität nicht im Mittelpunkt. Wir haben uns mit diesem Thema in einem anderen Beitrag beschäftigt und stellen fest, dass (i) die internationale und intertemporale Varianz der redistributiven Wirkungen von Austeritätspolitik empirisch gering ist, dass (ii) der Wohlfahrtsstaat im Zuge von Haushaltskonsolidierungen grundsätzlich nicht von Kürzungen verschont bleibt und dass (iii) die parteipolitische Zusammensetzung der Regierung hierbei weitgehend unerheblich ist. ${ }^{11}$

Wie sonst lässt sich der fehlende Einfluss des Parteienwettbewerbs auf Austerität erklären?

Zum einen ließe sich an der These der New Politics anknüpfen. Ihr zufolge sind die Logiken, die dem Abbau des Wohlfahrtsstaats zugrunde liegen, gänzlich von jenen der Ausbauphase verschieden. Da der Wohlfahrtstaat sich einer starken Unterstützung durch die Bevölkerung erfreue, gingen Regierungen das elektorale

9 Armingeon, K.: Austeritätspolitik: Was Parteien bewirken und Märkte mögen, in: Armingeon, K. (Hg.): Staatstätigkeiten, Parteien und Demokratie. Festschrift für Manfred G. Schmidt, Wiesbaden, 2013, 113 37; Wagschal, U./Wenzelburger, G.: Haushaltskonsolidierung, Wiesbaden, 2008; Wagschal, U./Wenzelburger, G.: When do Governments Consolidate? A Quantitative Comparative Analysis of 23 OECD Countries (1980-2005), in: Journal of Comparative Policy Analysis: Research and Practice, 14/1 (2012), 45-71.

10 Mierau, J.O./Jong-A-Pin, R./de Haan, J.: Do political variables affect fiscal policy adjustment decisions? New empirical evidence, in: Public Choice, 133 (2007), 297-319; Wenzelburger, G.: Interaktiv, indirekt und nur stellenweise: Wie Parteien Budgetkonsolidierungen beeinflussen, in: Zeitschrift für Politikwissenschaft, 19/4 (2009), 493-536; Hübscher, E./Sattler, T.: Fiscal Consolidation under Electoral Risk, unpubliziertes Manuskript, January 15, 2014, (2014).

11 Armingeon, K./Guthmann, K./Weisstanner, D.: Choosing the Path of Austerity: How Policy Coalitions Shape Welfare-Policy Choices in Periods of Fiscal Consolidations, a.a.O.. 
Risiko eines Wohlfahrtsstaatsabbaus gar nicht erst ein - insbesondere, wenn sie keine Chance sehen, ihre „Täterschaft“" zu verschleiern (blame avoidance). ${ }^{12}$ Dieses Argument allein kann aber nicht erklären, weshalb es dennoch zu Abbaupolitiken kommt und weshalb die dafür zuständigen Regierungsparteien nicht durchweg an der Wahlurne bestraft werden. ${ }^{13}$ Revisionen der New Politics-These weisen daher auf weitere mögliche Pfade zu Konsolidierungsmaßnahmen hin, die nicht bloß Ausprägungen von blame avoidance sind, sondern etwa offene Reformprozesse und Verhandlungen mit einer Vielzahl von Teilnehmern anstreben, mit dem Ziel von breiten Mehrheitskoalitionen, die der Minimierung des elektoralen Risikos dienen. ${ }^{14}$

Zum anderen könnte es sein, dass weder die Parteiendifferenz- noch die New Politics-Thesen die strategische Situation der beteiligten Akteure hinreichend berücksichtigen. Zunächst wird der funktionale Zwang unterschätzt: In Zeiten globalisierter Finanzmärkte und der europäischen Währungsunion haben viele Regierungen - unabhängig von ihrer ideologischen Orientierung - häufig keine andere Wahl als zu sparen. In dieser Situation könnte es von Vorteil sein, wenn sich ein Programm auf möglichst viele politische Akteure abstützen kann. Das hat nicht nur mit der gemeinsamen politischen Verantwortung zu tun, sondern auch mit der Erwartung, dass die Bestrafung an der Wahlurne milder ausfällt: Die Wählerinnen und Wähler sehen viele Parteien gemeinsam am Strick der Konsolidierung ziehen. Damit gibt es, abgesehen von extremistischen und populistischen Parteien, kaum verbleibende Wahlalternativen. In diesem Sinne ist „geteiltes elektorales Leid halbes Leid“, weil die Einschränkung der Wahlalternativen das Ausmaß der Stimmenverluste der Regierungsparteien verringert. Wenn alle wichtigen Akteure in das Reformprogramm eingebunden sind, kann sich zudem niemand mehr Vorteile verschaffen, indem er der Regierung den

12 Pierson, P.: Dismantling the Welfare State? Reagan, Thatcher and the Politics of Retrenchment, Cambridge, 1994; Pierson, P.: The New Politics of the Welfare State, in: World Politics, 48/2 (1996), 143-79.

13 Armingeon, K./Giger, N.: Conditional Punishment. A comparative analysis of the electoral consequences of welfare state retrenchment in OECD nations, 1980-2003, in: West European Politics, 31/3 (2008), 558-80; Giger, N.: The Risk of Social Policy? The Electoral Consequences of Welfare State Retrenchment and Social Policy Performance in OECD Countries, London and New York, 2011; Giger, N.: Is Social Policy Retrenchment Unpopular? How Welfare Reforms Affect Government Popularity, in: European Sociological Review, 28/5 (2012), 691-700; Giger, N./Nelson, M.: The Electoral Consequences of Welfare State Retrenchment: Blame Avoidance or Credit Claiming in the Era of Permanent Austerity?, in: European Journal of Political Research, 50/1 (2011), 1-23; Kersbergen, K.v./Vis, B.: Comparative Welfare State Politics. Development, Opportunities, and Reform, Cambridge, 2013; Vis, B.: Politics of Risk-taking. Welfare State Reform in Advanced Democracies, Amsterdam, 2010.

14 Levy, J.D.: Welfare Retrenchment, in: Castles, F.G./Leibfried, S./Lewis, J./Obinger, H./Pierson C. (Hg.): The Oxford Handbook of the Welfare State, Oxford, 2010, 552-565. 
Abbau des Wohlfahrtsstaates vorwirft. Diesem Argument liegt die Idee des circling the wagon der blame avoidance-Literatur zugrunde - eine Strategie der geteilten Verantwortlichkeit durch den Konsens breiter Koalitionen, um die unvermeidlichen Schuldvorwürfe für die unpopuläre Politik zu diffundieren. ${ }^{15}$

Ein weiterer Vorteil dieser Konstellation kommt hinzu: In einer Koalition aus wenigen Akteuren besteht die Neigung, die Kosten der Politik auf andere Gruppen zu externalisieren, was deren Opposition anfachen wird. Sind dagegen alle wichtigen Akteure in einer Reformkoalition versammelt, treten solch horizontale Umverteilungen kaum noch auf. Die Akteure wissen, dass sie und die von ihnen repräsentierten Gruppen ihren Teil zur Sanierung der Staatsfinanzen beitragen müssen, ohne dass ihre Kooperationsbereitschaft ausgebeutet werden könnte. Zudem können Sie auf eine vertikale Umverteilung in der Zeitdimension hoffen, über die sich ihr Opfer von heute in der Zukunft auszahlt. So können linke Parteien darauf setzen, dass eine Begrenzung der Ausgabendynamiken des Wohlfahrtsstaats zu seiner langfristigen Stabilität beiträgt. Dieses Argument ist jüngst von Jacobs entwickelt worden ${ }^{16}$ und kann sich auf eine ähnliche These über kooperative Vetopunkte stützen, ${ }^{17}$ welche letztlich auch den Theorien über Konsensusdemokratien zugrunde liegt: Breit abgestützte Reformkoalitionen sind besser als Regierungen in Mehrheitsdemokratien in der Lage, gemeinwohlverträgliche Politiken zu entwickeln und durchzusetzen. ${ }^{18}$ Dies gilt zunächst für Koalitionen zwischen Parteien (wie sie in unserer Arbeit im Fokus stehen), kann aber auch auf Interessengruppen erweitert werden. Regierungen werden schmerzvolle Konsolidierungsmaßnahmen eher in Angriff nehmen, wenn sie Interessenverbände an ihrer Seite wissen, die diese Politik im eigenen langfristigen Interesse unterstützen (vertikale Investition). Diese These deckt sich mit Befunden der älteren Korporatismusforschung ${ }^{19}$ und wird überzeugend durch die sozialpartnerschaftlich konzertierten Modernisierungen des Wohlfahrtsstaats in

15 Weaver, K.: The Politics of Blame Avoidance, in: Journal of Public Policy, 6/4 (1986), 371-398; König, P.D./Wenzelburger, G.: Toward a Theory of Political Strategy in Policy Analysis, in: Politics \& Policy, 42/3 (2014), 400-430.

16 Jacobs, A.M.: Governing for the Long Term. Democracy and the Politics of Investment., a.a.O.

17 Crepaz, M.M.L.: Constitutional structure and regime performance in 18 industrialized democracies: A test of Olson's hypothesis, in: European Journal of Political Research, 29/1 (1996), 87-104; Crepaz, M.M.L.: Inclusion versus Exclusion: Political Institutions and Welfare Expenditures, in: Comparative Politics, 31/1 (1998), 61-80.

18 Lijphart, A.: Patterns of Democracy: Government Form and Performance in Thirty-Six Countries. 2nd edition, New Haven, 2012.

19 Katzenstein, P.J.: Small States in World Markets. Industrial Policy in Europe, Ithaca/London, 1985; Katzenstein, P.J.: Small States and Small States Revisited, in: New Political Economy, 8/1 (2003), 930. 
Nordeuropa gestützt. ${ }^{20}$ Dieser Argumentation folgend ließe sich ein der Vetospielertheorie entgegengesetztes Ergebnis erwarten: Austeritätsprogramme von substantiellem Umfang sind umso wahrscheinlicher, auf je mehr Vetospieler sie sich stützen.

In diesem Beitrag beschränken wir uns bei der Messung von breiten Reformkoalitionen auf die Mehrheiten, die der Regierung im Parlament zur Verfügung stehen. Wir unterscheiden zwischen „breiten“ und „minimalen“ Koalitionen. Breite Reformkoalitionen sind unter ,großen Koalitionen“ und unter Minderheitsregierungen gegeben. Große Koalitionen sind surplus (,übergroße“) Koalitionen; also Regierungen, die sich aus mehr Parteien zusammensetzen, als für eine parlamentarische Mehrheit notwendig wären. Auch Minderheitsregierungen werden als breite Reformkoalitionen definiert, weil es in ihrem Wesen liegt, sich ihre Mehrheiten im Parlament fallweise über Konzessionen an NichtRegierungsparteien zu verschaffen. Um Unwägbarkeiten des parlamentarischen Abstimmungsprozesses zu vermeiden, besteht ein Anreiz, diese Mehrheiten möglichst groß zu gestalten. ${ }^{21} \mathrm{Zu}$ den minimalen Reformkoalitionen zählen wir Einparteienregierungen und Regierungskoalitionen, die nur gerade so viele Parteien auf sich vereinen, wie für eine parlamentarische Mehrheit mindestens nötig sind (minimal winning Koalitionen).

Wir argumentieren, dass die so definierte Breite einer Reformkoalition eine zentrale Determinante von Austeritätspolitik darstellt. Gleichzeitig unterstreichen wir jedoch, dass auch die traditionelleren Ansätze der Parteiendifferenzhypothese und der Vetospielertheorie einen substantiellen Beitrag zur Erklärung von Sparmaßnahmen leisten.

Im Kern geht unser Beitrag von der Annahme aus, dass, um die teils widersprüchlichen Erwartungen der verschiedenen Theoriestränge aufzulösen, zwingend zwischen der grundsätzlichen Entscheidung für ein Austeritätsprogramm

20 Anderson, K.M.: The Politics of Retrenchment in a Social Democratic Welfare State. Reform of Swedish Pensions and Unemployment Insurance, in: Comparative Political Studies, 34/9 (2001), 106391; Kuhnle, S.: The Scandinavian welfare state in the 1990s: Challenged but viable, in: West European Politics, 23/2 (2007), 209-28; Obinger, H./Starke, P./Moser, J.: Pioneers of Paradigmatic Change? Welfare State Transformation in Small Open Economies, Paper presented at the DVPW Kongress, Kiel, 2009; Starke, P./Kaasch, A./Van Hooren, F.: The Welfare State as Crisis Manager. Explaining the Diversity of Policy Responses to Economic Crisis, Houndsmills, Basingstoke und New York, 2013.

21 Lijphart, A.: Patterns of Democracy: Government Form and Performance in Thirty-Six Countries, a.a.O. Gleichzeitig unterscheiden sich Minderheitsregierungen von übergroßen Koalitionen darin, dass sie sich wechselnde Mehrheiten mit unterschiedlichen Partnern verschaffen können und unser Argument des ,geteilten elektoralen Leids“ nicht zwingend für sie relevant sein muss. Daher diskutieren wir in der empirischen Analyse alternativ eine getrennte Konzeptualisierung von Minderheitsregierungen und übergroßen Koalitionen. 
einerseits (als „erste Stufe“) und dessen Umfang andererseits (als „Zweite Stufe“) konzeptionell differenziert werden muss. Nur so können die relativen Einflüsse der verschiedenen Erklärungsgrößen präzise und vollständig erfasst werden. Dies liegt darin begründet, dass die genannten Phänomene (Auftretenswahrscheinlichkeit und Umfang von Austerität) von unterschiedlichen Faktoren geprägt, und vor allem in gegensätzlicher Richtung beeinflusst werden können. So kann sich eine Variable positiv auf die Wahrscheinlichkeit auswirken, dass ein Konsolidierungsprogramm überhaupt zustande kommt, aber gleichzeitig einen negativen Einfluss auf dessen Umfang haben. Dieser Möglichkeit muss sowohl konzeptionell als auch methodisch Rechnung getragen werden, selbst wenn sich der politische Entscheidungsprozess in der Realität nicht immer trennscharf in verschiedene Phasen zerlegen lässt.

In der Frage der Wahrscheinlichkeit, dass es überhaupt zu einem Konsolidierungsprogramm kommt (erste Stufe), sollte die Erklärungskraft der Parteiendifferenzhypothese am höchsten sein. Von rechten Parteien dominierte Regierungen sollten sowohl aufgrund ihrer ideologischen Orientierung, als auch den Interessen ihrer Wählerschaft folgend, einer restriktiven Fiskalpolitik grundsätzlich positiv gegenüberstehen. Von ihnen erwarten wir, dass sie eher ein Sparprogramm initiieren und zügig umsetzen können, als es für Parteien der politischen Linken/Mitte der Fall ist. Hieraus ergibt sich unsere erste empirisch überprüfbare Hypothese:

H1: Von rechten Parteien dominierte Regierungen weisen eine höhere Wahrscheinlichkeit auf, fiskalische Konsolidierungen durchzuführen.

Nun ist jedoch zu bedenken, dass rechte Parteien, die ein Sparprogramm initiieren, die Opposition der Wohlfahrtsstaatsparteien zu fürchten haben, nicht zuletzt weil sie geneigt sind, die Kosten der Anpassung asymmetrisch auf deren Klientel zu verschieben. Dies führt zur zweiten Stufe - der Frage nach dem Umfang des Konsolidierungsprogramms. Zwar sollten rechte Parteien gemäß Hypothese 1 überdurchschnittlich häufig ein Konsolidierungsprogramm initiieren - auch als Signal an die eigene Wählerschaft. Der Umfang der von ihnen umgesetzten Programme dürfte jedoch, um das Risiko einer Abstrafung an der Urne angesichts einer lautstarken Opposition aus Wohlfahrtsstaatsparteien, Gewerkschaften und den Begünstigten staatlicher Ausgabenprogramme zu minimieren, vergleichsweise klein ausfallen. Wenn ein Konsolidierungsprogramm einer rechten Regierung beschlossen ist, gibt es für diese Opposition kaum Anreize zur Teilung der politischen Verantwortung. Der Widerstand dürfte mit dem Ausmaß der Konsolidierung wachsen, weshalb rechte Regierungen ihre Sparprogramme in ihrem 
eigenen elektoralen Interesse von vergleichsweise geringem Umfang gestalten sollten. Ein von linken/zentristischen Parteien initiiertes Sparprogramm sollte dagegen zwar grundsätzlich eine seltenere Erscheinung sein (H1). Wenn es aber schließlich dazu kommt, sollte es vergleichsweise umfangreich ausfallen. Erstens sind die grundsätzlichen ideologischen und programmatischen Hürden, die sich linken und zentristischen Parteien und der durch sie repräsentierten Wählerklientel auf ihrem Weg zur Austerität üblicherweise in den Weg stellen, bereits genommen: Die prinzipielle Entscheidung für ein Sparprogramm ist getroffen, die relevanten Akteure sind von der Notwendigkeit einer Konsolidierung überzeugt. Zweitens ist von den rechten Parteien und ihrer Wählerschaft zumindest inhaltlich in der Regel kein allzu scharfer Widerspruch zu erwarten. Drittens genießen linke/zentristische Parteien eher das Vertrauen der Wohlfahrtsstaatsklientel, die Austerität sozialverträglich (und womöglich stärker einnahmebasiert) zu gestalten. Der letzte Punkt beschreibt ein Nixon-goes-to-China-Szenario: Aufgrund ihrer historischen Rolle als Begründer und Garanten des Wohlfahrtsstaates sind linke/zentristische Parteien eher in der Lage, ihn im Zuge von Sparmaßnahmen umfassend zu reformieren. ${ }^{22}$ Dies führt zur zweiten Hypothese:

H2: Wenn von rechten Parteien dominierte Regierungen fiskalische Konsolidierungen durchführen, fallen diese im Umfang kleiner aus als jene von Regierungen einer anderen parteipolitischen Färbung.

Wie oben dargelegt, sollte auch die Breite einer Reformkoalition eine zentrale Determinante von Austeritätspolitik sein. Für die Phase der prinzipiellen Entscheidung zur Austerität erwarten wir von ihr eine Wirkung im Sinne der klassischen Vetospielertheorie - und damit einen negativen Einfluss. In dieser Phase geht es um die grundsätzliche Frage, ob der Status Quo mit einem Konsolidierungsprogramm verändert werden soll oder nicht. Abstufungen zwischen diesen Extrempositionen - z.B. in Form von Kompromissen hinsichtlich des Umfangs oder der konkreten Ausgestaltung der Maßnahmen - sind (noch) nicht möglich. Sowohl unter breiten als auch unter minimalen Regierungskoalitionen ist der Konsens zwischen allen beteiligten Akteuren die zentrale Voraussetzung für die Initiierung eines Konsolidierungsprogramms. Unter breiten Koalitionen ist dies jedoch im Sinne Tsebelis ${ }^{23}$ prinzipiell ein besonders zeitaufwendiger, störungsund blockadeanfälliger Prozess, da sich die vielfältigen, oft sehr weit auseinanderliegenden, Interessen sämtlicher in der Regierungskoalition repräsentierten 
Wählergruppen nur schwer ,unter einen Hut“ bringen lassen. Die Wahrscheinlichkeit, dass eine Koalition Akteure enthält, die auch nur den leisesten Anschein von Austerität aus ideologischen oder politischen Gründen kompromisslos ablehnen und die Regierungskoalition durch die von ihnen mobilisierte Opposition ins Wanken bringen (oder zumindest schrumpfen lassen), nimmt mit wachsender Größe zu. Hierbei spielt auch eine Rolle, dass eine breite Koalition kaum Möglichkeiten zur Externalisierung der mit der Konsolidierung verbundenen Kosten hat. Gerade jene Parteien, deren Wählerpotential sich primär aus ihrer Opposition zur Austerität speist, würden durch das Mittragen dieser bei ihren Wählern verhassten Politik ihre Existenzberechtigung verlieren.

Minimale Koalitionen haben es dagegen leichter, in der Frage der prinzipiellen Notwendigkeit eines Konsolidierungsprogramms einen Konsens herbeizuführen, nicht zuletzt da sich für sie mehr Möglichkeiten zur Externalisierung der damit verbundenen Kosten bieten. Dies führt zu unserer dritten Hypothese:

H3: Breite Reformkoalitionen weisen eine niedrigere Wahrscheinlichkeit auf, fiskalische Konsolidierungen durchzuführen als minimale Reformkoalitionen.

Für Koalitionen, in denen sich alle beteiligten Akteure grundsätzlich einig sind, dass ein Konsolidierungsprogramm initiiert werden soll, erwarten wir für die Breite dieser Koalition nun einen gegenteiligen, positiven Effekt auf den Umfang des Sparprogramms. Konzeptionell befinden wir uns damit wieder auf der zweiten Stufe des politischen Entscheidungsprozesses. In dieser Phase steht die Austeritätspolitik nicht (mehr) grundsätzlich zur Disposition - nur bezüglich des Umfangs und der konkreten Ausgestaltung der Maßnahmen ist (noch) ein Konsens zwischen den beteiligten Akteuren herbeizuführen. An dieser Stelle wird die bisherige Schwäche der breiten Koalition (auf der ersten Stufe) zur Stärke (auf der zweiten Stufe): Da eine breite Mehrheit von Wählergruppen in der Koalition repräsentiert sind, ist kein umfassender gesellschaftlicher Widerstand gegen die Sparmaßnahmen zu erwarten. Eine Externalisierung der Kosten ist bei breiten Koalitionen zwar prinzipiell nur begrenzt möglich - dies sollte aber auch nur noch eine begrenzte Rolle spielen: Zum einen steht die grundsätzliche Notwendigkeit einer Konsolidierung innerhalb der Koalition nicht zur Debatte, sodass angesichts der limitierten Externalisierungsmöglichkeiten eine gewisse Bereitschaft vorhanden sein dürfte, die Kosten selbst zu tragen; zum anderen lässt sich ein Kostenausgleich innerhalb der Koalition über Kompromisse hinsichtlich der konkreten Ausgestaltung von Einzelmaßnahmen erreichen. 
Minimale, zur Konsolidierung entschlossene Koalitionen stützen sich dagegen per Definition nur auf eine kleine gesellschaftliche Mehrheit und sehen sich damit potentiell einer breiten Anti-Austeritäts-Opposition gegenüber. Diese Opposition sollte umso schärfer ausfallen, je umfangreicher das Konsolidierungsprogramm ausfällt. Hinzu kommt, dass minimale Koalitionen die Möglichkeit - und damit in der Regel auch einen Anreiz - haben, die Kosten der Austerität zu externalisieren. Dieser Externalisierung sind jedoch Grenzen gesetzt, um die Opposition der nicht repräsentierten Interessen nicht weiter anzufachen und keine elektorale Risiken einzugehen, was sich ebenfalls negativ auf den Umfang der Sparmaßnahmen auswirken sollte.

In der Gesamtsicht erwarten wir demnach, dass sich Tsebelis' parteipolitische Vetospieler der ersten Stufe, wenn sie sich grundsätzlich zur Austerität bekannt haben, in der Frage nach dem Ausmaß der Konsolidierung auf der zweiten Stufe eher im Sinne der kooperativen Vetopunkte nach Crepaz ${ }^{24}$ verhalten. Deshalb werden fiskalische Konsolidierungen unter breiten Koalitionen zwar seltener stattfinden, in ihrem Umfang jedoch größer sein. Dies führt zur vierten Hypothese:

H4: Wenn breite Reformkoalitionen fiskalische Konsolidierungen durchführen, fallen diese im Umfang größer aus als jene von minimalen Reformkoalitionen.

Interpretiert man nun alle vier Hypothesen als Ganzes (vgl. Tab 1), sollte sich für die erste Stufe folgendes Bild ergeben: (1) Unter rechten Parteien in minimalen Reformkoalitionen sind fiskalische Konsolidierungen - genau wie es die Parteiendifferenzthese in Verbindung mit der Vetospielertheorie vermuten lässt - am wahrscheinlichsten; (2) etwas weniger wahrscheinlich sind Austeritätsprogramme unter rechten Parteien in breiten Reformkoalitionen sowie unter zentristischen/linken Parteien in minimalen Reformkoalitionen; (3) unter zentristischen/linken Regierungen in breiten Reformkoalitionen sind Sparprogramme schließlich - wieder im Einklang mit der Parteiendifferenzthese in Verbindung mit der Vetospielertheorie - am unwahrscheinlichsten.

Für die zweite Stufe des Umfangs der Konsolidierung erwarten wir spiegelbildlich dazu das folgende Muster: (1) Unter linken/zentristischen Regierungen in breiten Reformkoalitionen sind Sparpakete am größten, im Einklang mit dem

24 Crepaz, M.M.L.: Constitutional structure and regime performance in 18 industrialized democracies: A test of Olson's hypothesis, a.a.O.; Crepaz, M.M.L.: Inclusion versus Exclusion: Political Institutions and Welfare Expenditures, a.a.O. 
von Kitschelt beschriebenen Nixon-goes-to-China-Szenario und im Sinne der kooperativen Vetopunkte nach Crepaz oder der Argumentation von Jacobs; (2) etwas kleiner sind die Sparprogramme unter rechten Regierungen in breiten Reformkoalitionen sowie unter linken/zentristischen Regierungen in minimalen Reformkoalitionen; (3) unter rechten Regierungen in minimalen Reformkoalitionen sind die Konsolidierungspakete am kleinsten, weil für sie die Wahrscheinlichkeit am größten ist, ins wahlpolitische Sperrfeuer einer großen und üblicherweise durch linke Parteien gut organisierten politischen und gesellschaftlichen Opposition zu geraten.

Tabelle 1: Übersicht der Hypothesen zu den erwarteten Parteien- und Koalitionseffekten

1. Stufe: Eintrittswahrscheinlichkeit der Konsolidierung:

\begin{tabular}{l|cc} 
& Minimale Koalitionen & Breite Koalitionen \\
\hline Rechte Regierungen & + & $+/-$ \\
Nicht-rechte Regierungen & $+/-$ & -
\end{tabular}

2. Stufe: Umfang der Konsolidierung:

\begin{tabular}{l|cc} 
& Minimale Koalitionen & Breite Koalitionen \\
\hline Rechte Regierungen & - & $+/-$ \\
Nicht-rechte Regierungen & $+/-$ & +
\end{tabular}

Wir mobilisieren Evidenz für unsere Hypothesen auf Basis deskriptiver Statistiken sowie mittels systematischer Hypothesentests in einer Reihe multivariater Regressionsmodelle unter Einbezug politischer und ökonomischer KontrollgröBen.

\section{Daten}

Fiskalische Konsolidierungen werden in den Wirtschaftswissenschaften und der politischen Ökonomie in der Regel über die jährlichen Veränderungsraten der Bilanz des Staatshaushaltes, oder die um Zinszahlungen bereinigte Primärbilanz, gemessen. ${ }^{25}$ Um Konjunkturschwankungen auszublenden, wird häufig die zyklisch korrigierte Variante dieses Indikators, die cyclically adjusted primary balance (CAPB) verwendet. Verbessert sich die CAPB über einen bestimmten

25 Wagschal, U./Wenzelburger, G.: Haushaltskonsolidierung, a.a.O; Wagschal, U./Wenzelburger, G.: When do Governments Consolidate? A Quantitative Comparative Analysis of 23 OECD Countries (1980-2005), a.a.O. 
Schwellenwert - bspw. 1,5\% des BIP ${ }^{26}$ - hinaus, wird von einer Konsolidierung gesprochen.

In der neueren Literatur wird die Validität und Reliabilität dieser Operationalisierung über Veränderungen der Primärbilanz oder der CAPB jedoch angezweifelt. Zunächst setzten beide Indikatoren beim Ergebnis einer Konsolidierung an, anstatt bei der theoretisch oft stärker im Fokus stehenden Konsolidierungspolitik, welche dieses Ergebnis zwar ausgelöst haben kann, aber nicht zwangsläufig ausgelöst hat. Daher wird die Verwendung der CAPB auch als Mittel verstanden, diskretionäre Politikveränderungen aus den Daten herauszufiltern - und den Indikator somit um all jene Anteile zu bereinigen, die nicht auf solche Politikänderungen zurückgehen. Selbst wenn dies gelingt, bleiben die tatsächlich hinter den verbleibenden Werten stehenden politischen Motivationen jedoch letztlich unberücksichtigt.

Doch es kommen weitere Probleme hinzu: Die Methoden zur zyklischen Korrektur der Primärbilanz unterscheiden sich nicht nur erheblich über verschiedene Datenanbieter (z.B. OECD, IMF) hinweg, sondern leiden mitunter auch unter systematischen Messfehlern, die mit ökonomischen Entwicklungen korrelieren. ${ }^{27}$ In der Gesamtsicht ist die Validität solch ergebnisbasierter Indikatoren zur Messung von politischen Programmen der fiskalischen Konsolidierung somit fraglich.

Ein neuerer Ansatz zur Messung von Austeritätpolitik setzt daher unmittelbar an den von Regierung und Parlament verabschiedeten politischen Konsolidierungsprogrammen an, anstatt diese über die zyklische Korrektur von Ergebnisdaten zu approximieren. So identifizieren Devries et al. ${ }^{28}$ in ihrem handlungsorientierten (action-based) Ansatz mittels historischer Dokumente (wie Budget- und andere Regierungspublikationen, Zentralbankberichte, Berichte internationaler Organisationen etc.) für 17 OECD-Staaten zwischen 1978 und 2009 all jene Politikmaßnahmen, deren ausdrückliche Motivation die fiskalische Konsolidierung war. Die fiskalischen Auswirkungen dieser Veränderungen in Staatseinnahmen und -

26 Alesina, A./Ardagna, S.: Large Changes in Fiscal Policy: Taxes versus Spending, in: NBER Working Paper 15438 (2009).

27 Devries, P./Guajardo, J./Leigh, D./Pescatori, A.: A New Action Based Dataset of Fiscal Consolidation, in: IMF Working Paper, WP/11/128 (2011); IMF: World Economic Outlook. Recovery, Risk and Rebalancing. October 2010., Washington, D.C., 2010, 96; Wagschal, U./Wenzelburger, G.: When do Governments Consolidate? A Quantitative Comparative Analysis of 23 OECD Countries (1980-2005), a.a.O.

28 Devries, P./Guajardo, J./Leigh, D./Pescatori, A.: A New Action Based Dataset of Fiscal Consolidation, a.a.O. 
ausgaben wurden in Prozent des BIP unter der Annahme quantifiziert, dass alle anderen Umstände (z.B. externe Krisen oder die Wirkungen der automatischen Stabilisatoren) konstant bleiben. In dem daraus resultierenden Datensatz von 544 Länderjahren werden insgesamt 173 Beobachtungen als Jahre fiskalischer Konsolidierung eingestuft.

Unsere abhängige Variable basiert auf diesen Daten von Devries et al., ${ }^{29}$ welche wir jedoch in zweierlei Hinsicht modifiziert haben. Zum einen umfasst der Originaldatensatz auch solche Sparmaßnahmen, die sich nur vorrübergehend oder einmalig auf das Haushaltsdefizit auswirken. Dazu gehören z.B. eine temporäre Erhöhung der Mehrwertsteuer oder der Verkauf von Staatseigentum. Da unser theoretisches Interesse in erster Linie den Bestimmungsfaktoren von Austeritätspolitik gilt, die strukturelle, permanente Veränderungen, bspw. an wohlfahrtsstaatlichen Institutionen, vornimmt, haben wir unsere Daten um derartige temporäre und einmalige Maßnahmen bereinigt. ${ }^{30}$

Zum anderen haben wir die Daten für mehrjährige Konsolidierungsphasen, während denen ein Regierungswechsel stattfand, dahingehend modifiziert, dass die spezifischen Sparmaßnahmen derjenigen Regierung zugeordnet wurden, die sie verabschiedet hat, anstatt jener, die in späteren Jahren ausschließlich für die Implementation zuständig war. Aufgrund dieser Modifikationen reduziert sich die Zahl der von uns analysierten Konsolidierungsjahre auf $163 .{ }^{31}$ Des Weiteren verkleinert sich unsere Fallzahl für die multivariate Analyse wegen fehlender Daten für zentrale erklärende Variablen nochmals auf schließlich 159 Konsolidierungsjahre von insgesamt 527 Länderjahren.

Unsere Untersuchung beinhaltet zwei Haupterklärungsfaktoren: Erstens die Breite der Reformkoalition, auf die sich eine Regierung im Parlament stützen kann; und zweitens die parteipolitische Zusammensetzung der Regierung. ${ }^{32}$

Wie oben diskutiert, unterscheiden wir hinsichtlich der Breite einer Reformkoalition zwischen breiten und minimalen Regierungskoalitionen. Für deren Messung verwenden wir eine Dummy-Variable mit dem Wert 1 für übergroße (surplus) Koalitionen sowie für Minderheitsregierungen. Einparteienregierungen und $m i$ nimal winning Koalitionen werden mit dem Wert 0 kodiert.

\footnotetext{
A.a.O.

Die Ergebnisse mit den unveränderten Daten von Devries et al. bleiben substantiell identisch.

Details zu den Modifikationen sind auf Anfrage verfügbar.

32 Die Daten für beide Variablen stammen aus Armingeon, K./Knöpfel, L./Weisstanner, D./Engler, S./Potolidis, P./Gerber, M.: Comparative Political Data Set I 1960-2011, a.a.O.
} 
Bei der parteipolitischen Zusammensetzung der Regierung konzentrieren wir uns auf die Unterscheidung zwischen rechten (überwiegend konservative und liberale) und „nicht-rechten“ Parteien. Zur Messung verwenden wir ebenfalls eine Dummy-Variable, die den Wert 1 annimmt, wenn als rechts klassifizierte Parteien in einem bestimmten Länderjahr mehr als $50 \%$ der Kabinettsposten auf sich vereinen konnten - und die Regierung somit dominierten. Linke, zentristische und gemischte Regierungen werden mit 0 kodiert und bilden die Referenzgruppe. ${ }^{33}$

In unserer statistischen Analyse berücksichtigen wir die folgenden Kontrollvariablen. Für den Einfluss ökonomischer Bestimmungsgrößen kontrollieren wir einerseits mit der Arbeitslosenrate des Vorjahres und andererseits mit einem Maß des fiskalischen Problemdrucks des Vorjahres, welches die wichtigsten ökonomischen Koordinaten fiskalischer Stabilität - die Höhe der Staatsverschuldung, die Zinshöhe, die Höhe des Haushaltsdefizits sowie die Wachstumsrate des BIP - in einem einzigen Indikator vereint. ${ }^{34}$ Gegenüber der Verwendung von Einzelvariablen im Regressionsmodell liefert diese dynamische Maßzahl ein weitaus präziseres Bild der langfristigen Nachhaltigkeit öffentlicher Finanzen und der sich daraus ergebenden funktionalen Zwänge. ${ }^{35}$ Aufgrund der statistischen Kontrolle dieser Variable können wir Endogenität - bspw. im Sinne eines systematisch geringeren fiskalischen Problemdrucks unter breiten Koalitionen - ausschließen.

Für die Position im Wahlzyklus, auch in Interaktion mit der Knappheit der Wahl, kontrollieren wir, da wir im Anschluss an Hübscher und Sattler ${ }^{36}$ davon ausgehen, dass bei prognostiziertem knappen Wahlausgang das elektorale Risiko einer Konsolidierung weniger häufig eingegangen wird; und falls doch, dann eher zu Beginn einer Legislaturperiode. Wir operationalisieren dies über die Anzahl der Jahre seit der letzten Wahl (wobei das Wahljahr zur vorherigen Periode gezählt wird), den Abstand (in Stimmenprozenten) zwischen der größten und der zweit-

33 Die alternative Verwendung von Dummy-Variablen für von linken oder zentristischen Parteien dominierte Regierungen in der empirischen Analyse lieferte keine konsistenten oder statistisch signifikanten Ergebnisse. Eine kontinuierliche Kodierung von rechten Regierungen über den Anteil rechter Parteien an der Gesamtzahl der Kabinettsposten ändert nichts an den in diesem Beitrag vorgestellten Befunden.

34 Wir berechnen den fiskalischen Problemdruck, welcher die Veränderung der Staatsschuldenquote misst, als: Problemdruck $\mathrm{t}_{\mathrm{t}-1}=$ Staatsverschuldung $_{\mathrm{t}-2} *\left(\right.$ Zinssatz $\left._{\mathrm{t}-1}-\Delta \mathrm{BIP}_{\mathrm{t}-1}\right)+$ Defizit $_{\mathrm{t}-1}$, im Anschluss an Bénassy-Quéré, A./Coeuré, B./Jacquet, P./Pisani-Ferry, J.: Economic Policy, Theory and Practice, 2nd ed., New York, 2010, 168.

35 Turner, D./Spinelli, F.: Interest-rate-growth differentials and government debt dynamics, in: OECD Journal: Economic Studies, 1 (2012).

36 Hübscher, E./Sattler, T.: Fiscal Consolidation under Electoral Risk, a.a.O. 
größten Partei im Parlament (als Maß für den Parteienwettbewerb ${ }^{37}$ ) sowie über eine Interaktion zwischen beiden Variablen. Der Index institutioneller Beschränkungen von Schmidt $^{38}$ kontrolliert für die unterschiedlichen institutionellen Handlungsspielräume der Zentralregierung. ${ }^{39}$ Schließlich folgen wir den Empfehlungen von Carter und Signorino ${ }^{40}$ und fügen, um der temporalen Dimension der Daten gerecht zu werden, noch einen Zeit-Zähler, gemessen als die Anzahl der Jahre seit dem letzten Konsolidierungsereignis, sowie dessen quadrierte und kubische Transformation zu unseren statistischen Modellen hinzu.

\section{Deskriptive Analyse}

Abbildung 1 veranschaulicht die deskriptiven Befunde, wobei die oberen drei Säulendiagramme die Häufigkeit und die unteren drei Diagramme den Umfang fiskalischer Konsolidierungen, jeweils differenziert nach parteipolitischer Färbung und Koalitionstyp, illustrieren. Auf rechte Regierungen entfallen absolut gesehen die meisten Konsolidierungen (55 von 163 Konsolidierungsjahren im Datensatz, im Vergleich zu 108 unter ,nicht-rechten“ - davon 45 unter linken, 44 unter zentristischen und 19 unter gemischten Regierungen). Relativ zur Gesamtzahl aller rechten Regierungen führen allerdings nur drei von zehn eine Konsolidierung durch und unterscheiden sich somit nicht systematisch von den nichtrechten Parteien (linkes oberes Säulendiagramm in der Abbildung). Auf Basis dieser rein deskriptiven bivariaten Analyse finden wir also keine Unterstützung für unsere erste Hypothese.

Von den 163 Konsolidierungsjahren entfallen absolut gesehen 114 auf minimale und 49 auf breite Koalitionen (mittleres oberes Säulendiagramm). Im Einklang mit unseren theoretischen Erwartungen bestätigt auch der Blick auf die relative Häufigkeit dieses Muster: Während gut $35 \%$ aller minimalen Koalitionen ein Konsolidierungsprogramm initiieren, sind es unter den breiten Koalitionen nur etwa $20 \%$.

37 Siehe z.B. Bühlmann, M./Freitag, M.: Individual and Contextual Determinants of Electoral Participation, in: Swiss Political Science Review, 12/4 (2006), 13-47.

38 Schmidt, M.G.: When Parties Matter: A Review of the Possibilities and Limits of Partisan Influence on Public Policy, in: European Journal of Political Research, 30/2 (1996), 155-83.

39 Die Daten für sämtliche Kontrollvariablen stammen ebenfalls aus Armingeon, K./Knöpfel, L./Weisstanner, D./Engler, S./Potolidis, P./Gerber, M.: Comparative Political Data Set I 1960-2011, a.a.O.

40 Carter, D.B./Signorino, C.S.: Back to the Future: Modeling Time Dependence in Binary Data, in: Political Analysis, 18/3 (2010), 271-92. 
Beschränkt man sich ausschließlich auf Konsolidierungsprogramme unter rechten Regierungen (rechtes oberes Säulendiagramm), lässt sich zwischen breiten und minimalen Koalitionen jedoch kein systematischer Unterschied in der relativen Häufigkeit mehr feststellen. Die für den Gesamtdatensatz erkennbare unterschiedliche Konsolidierungsneigung der Koalitionstypen (mittleres oberes Säulendiagramm) beschränkt sich also offenbar ausschließlich auf die nicht-rechten Regierungen (wo fast $40 \%$ der minimalen, aber weniger als $20 \%$ der breiten Koalitionen ein Sparprogramm anstoßen) - und wird von diesen getrieben (rechtes oberes Säulendiagramm).

Abbildung 1:Relative Häufigkeit und durchschnittliche Größe fiskalischer Konsolidierungen nach parteipolitischer Färbung und Koalitionstyp
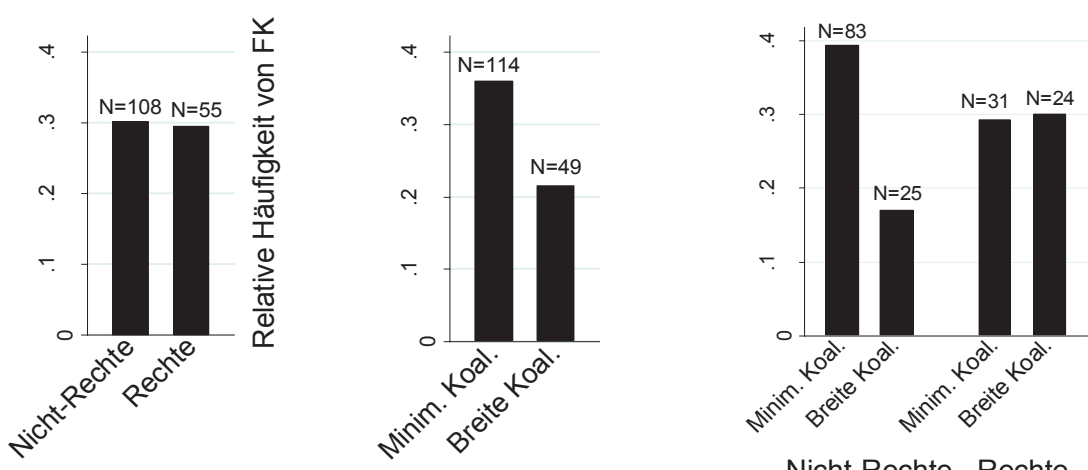

Nicht-Rechte Rechte
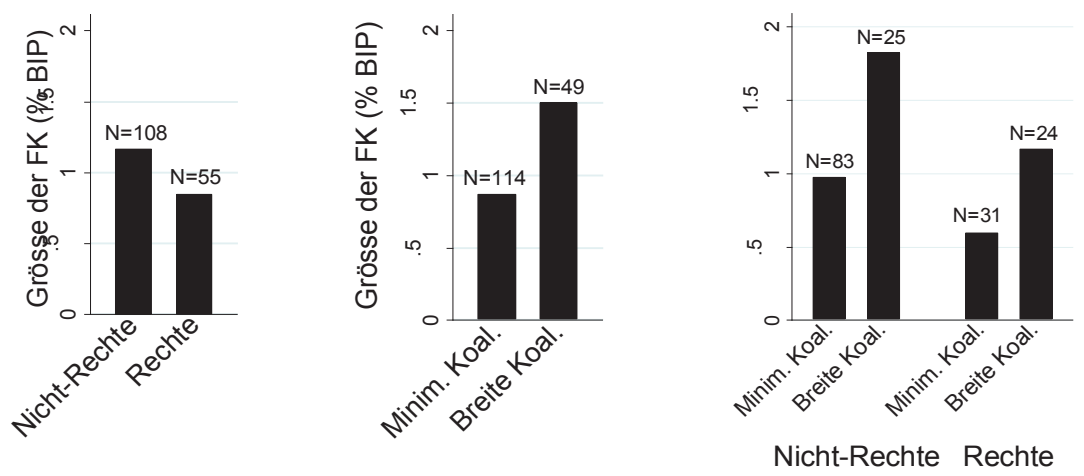

Anmerkung: Die relativen Häufigkeiten zeigen die Eintrittswahrscheinlichkeit einer fiskalischen Konsolidierung unter bestimmten Gruppen von Regierungen (rechte/nicht-rechte, minimale/breite) und berechnen sich aus der Anzahl der Konsolidierungen im Verhältnis zur Gesamtzahl dieser Regierungen im Datensatz. 
Bezüglich des Ausmaßes der Austerität - gemessen als erwartete Budgetveränderung in Prozent des BIP - ergibt sich ein entgegengesetztes Bild: Zwar initiieren breite Koalitionen, wie oben gezeigt, seltener ein Konsolidierungsprogramm; wenn sie es jedoch tun, fällt es im Durchschnitt größer aus, als unter minimalen Koalitionen (mittleres unteres Säulendiagramm).

Des Weiteren weisen die von rechten Regierungen durchgeführten Sparprogramme einen im Durchschnitt etwas geringeren Umfang auf als jene der NichtRechten. Dieser Größenunterschied hängt nicht vom Koalitionstyp ab - er gilt sowohl für breite als auch für minimale Koalitionen (rechtes unteres Säulendiagramm).

Schließlich hängt analog dazu auch der Einfluss des Koalitionstyps - im Gegensatz zur Phase der Initiierung von Austeritätspolitiken - nicht von der parteipolitischen Färbung einer Regierung ab: Der positive Einfluss breiter Koalitionen auf den Umfang eines Konsolidierungsprogramms gilt für rechte und nicht-rechte Regierungen gleichermaßen (rechtes unteres Säulendiagramm).

Auf Basis dieser deskriptiven Auswertung werden unsere Hypothesen zusammenfassend also weitgehend, aber nicht vollständig, bestätigt. Bevor wir die Ergebnisse näher diskutieren, unterziehen wir unsere Hypothesen im Folgenden einer systematischen statistischen Analyse unter Kontrolle alternativer Einflussfaktoren.

\section{Methode}

Wir interessieren uns für die Bestimmungsfaktoren des Auftretens fiskalischer Konsolidierungen sowie des Umfangs der Konsolidierungen. Wie bereits dargelegt, haben wir es also mit einem zweistufigen Prozess zu tun: erstens die Wahrscheinlichkeit, dass ein Konsolidierungsereignis eintritt (erste Stufe); und zweitens der erwartete Umfang des Sparpakets, falls das Ereignis eintritt (zweite Stufe). Beim Umfang der Konsolidierung handelt es sich somit um eine limitierte abhängige Variable, die einen positiv kontinuierlichen Wert annimmt, falls das Ereignis eintritt; und den Wert 0, falls es nicht eintritt. Aus methodischer Perspektive sind die Daten somit ,zensiert“: Wenn ein Konsolidierungsereignis nicht tatsächlich stattgefunden hat, kann nicht beobachtet werden, welchen Umfang es gehabt hätte, wenn es initiiert worden wäre. In diesem Fall liefert die 
Parameterschätzung mittels der Methode der kleinsten Quadrate (OLS) verzerrte Resultate. $^{41}$

Da die Werte der erklärenden Variablen für alle Beobachtungen verfügbar sind, kann in einem ersten Schritt die Wahrscheinlichkeit bestimmt werden, dass eine bestimmte Beobachtung in den nicht-zensierten Wertebereich fällt. Eine solche Lösung bietet das Tobit-Modell. ${ }^{42}$ Die Einschränkung des Tobit-Modells ist jedoch, dass die beiden geschätzten Parametervektoren - die Wahrscheinlichkeit, dass eine Beobachtung über die Zensurschwelle tritt und der Erwartungswert der Beobachtung, falls die Schwelle überschritten wurde - in einem fixen Verhältnis zueinander stehen. ${ }^{43}$ Mit anderen Worten kann eine bestimmte unabhängige Variable nicht gleichzeitig einen positiven Einfluss auf die Wahrscheinlichkeit des Auftretens eines Konsolidierungsereignisses und einen negativen Einfluss auf den Umfang des Sparprogramms ausüben. Schon auf Basis unserer theoretischen Argumentation ist ein klassisches Tobit-Modell für unsere Forschungsanlage somit ungeeignet. Dies bestätigen auch die Resultate der empirischen Analysen, wie weiter unten gezeigt wird. Aufgrund ihrer Flexibilität besser geeignet sind sogenannte „Hürden“-Modelle, die in der Lage sind, für (a) die Wahrscheinlichkeit des Eintretens der Konsolidierung und (b) ihren Umfang, falls sie eingetreten ist, unterschiedliche Parameter zu schätzen.

Wir verwenden ein Cragg-Modell, ${ }^{44}$ bei dem ein solches zweistufiges Modell geschätzt wird. Das Cragg-Modell ist eine natürliche Erweiterung des TobitModells, mit dem einzigen Nachteil leichter Effizienzverluste gegenüber der Tobit-Spezifikation. ${ }^{45}$ Zuerst bestimmt ein Probit-Modell für binäre abhängige Variablen die Wahrscheinlichkeit, dass ein Konsolidierungsereignis eintritt, d.h. die bei 0 liegende Schwelle des Nichteintretens überschritten wird. Im zweiten Schritt wird ein ,gestutztes“ Regressionsmodell (truncated regression) für den Erwartungswert des Konsolidierungsumfangs (gegeben die abhängige Variable ist $>0)$ geschätzt. Die beiden Stufen des Cragg-Modells können separat ge-

41 Long, S.J.: Regression Models for Categorical and Limited Dependent Variables, Thousand Oaks, CA, 1997, Kapitel 7; Tobin, J.: Estimation of Relationships for Limited Dependent Variables, in: Econometrica, 26/1 (1958), 24-36, hier 25.

42 Tobin, J.: Estimation of Relationships for Limited Dependent Variables, a.a.O.

43 Smith, D.A./Brame, R.: Tobit Models in Social Science Research, in: Sociological Methods \& Research, 31/3 (2003), 364-88, hier 366; Wooldridge, J.M.: Introductory Econometrics, A Modern Approach, 4th ed., Andover, 2009, 595.

44 Cragg, J.G.: Some Statistical Models for Limited Dependent Variables with Application to the Demand for Durable Goods, in: Econometrica, 39/5 (1971), 829-44.

45 Smith, D.A./Brame, R.: Tobit Models in Social Science Research, a.a.O., 367; Wooldridge, J.M.: Econometric Analysis of Cross Section and Panel Data, 2nd ed., Cambridge/London, 2010, 692-694. 
schätzt werden. ${ }^{46}$ Das Probit-Modell für binäre abhängige Variablen ist gegeben als:

$$
\operatorname{Pr}(\text { Konsolidierung }=1 \mid \mathrm{X})=\Phi(\mathrm{X} \beta)
$$

Der Erwartungswert des gestutzten Regressionsmodells ist gegeben als:

$$
\mathrm{E} \text { (Konsolidierung } \mid \text { Konsolidierung }>0, X)=X \beta+\sigma \lambda
$$

wobei X $\beta$ jeweils den Koeffizientenvektor der unabhängigen Variablen des Modells darstellt. Der Term $\lambda$ ist die inverse Mills ratio und korrigiert für die Stutzung der Daten - je größer die Stutzung, desto größer $\lambda$; $\sigma$ ist die Standardabweichung des unbekannten Fehlerterms in einem Modell mit vollständigen Daten. ${ }^{47}$ Das gestutzte Regressionsmodell ergänzt ein lineares Standardregressionsmodell somit um eine Korrektur für den fehlenden Teil der Daten. ${ }^{48}$

Eine potentielle Schwäche des Cragg-Modells liegt in der Annahme, dass die beiden Stufen des Modells unabhängig voneinander sind und durch separate Wahrscheinlichkeitsfunktionen geschätzt werden können. Eine Korrelation zwischen der Wahrscheinlichkeit einer Konsolidierung und des erwarteten Umfangs der Konsolidierung - hervorgerufen bspw. durch eine nicht im Modell enthaltene Drittvariable - ist jedoch denkbar. Als Robustheitstest ergänzen wir unsere Analyse daher um ein Heckman-Modell, ${ }^{49}$ welches das Problem der Selektionsverzerrung adressiert und ebenfalls zwischen Selektions- und Ergebnisprozess unterscheidet. Die im oben gezeigten gestutzten Regressionsmodell enthaltene inverse Mills ratio $(\lambda)$ wird dabei erneut zunächst mit einem Probit-Modell geschätzt und in der zweiten Stufe als unabhängige Variable in eine OLSRegression aufgenommen. Dadurch modelliert das Heckman-Modell die Korrelation zwischen den beiden Stufen. Weil die Schätzung unpräzise wird, wenn alle in der Selektionsgleichung enthaltenen Variablen auch in der Ergebnisgleichung

46 Breen, R.: Regression Models. Censored, Sample Selected, or Truncated Data, Thousand Oaks, CA, 1996, 34; Burke, W.J.: Fitting and Interpreting Cragg's Tobit Alternative Using Stata, in: Stata Journal, 9/4 (2009), 584-92.

47 Long, S.J.: Regression Models for Categorical and Limited Dependent Variables, a.a.O., 192-194; Breen, R.: Regression Models. Censored, Sample Selected, or Truncated Data, a.a.O, 46-47.

48 Die Verteilungsannahme des Cragg-Modells ist eine Normalverteilung der (latenten) abhängigen Variablen. Diese Annahme kann jedoch nicht getestet werden, da die Variable unvollständig - d.h. nur bei Werten über 0 - beobachtet wird. Wir haben die gestutzten Regressionsmodelle in Stata 13 mit der „truncreg“-Prozedur berechnet. Alternative Schätzungen mit einfacher OLS-Regression (für Werte grösser als null) ergeben substantiell identische Resultate.

49 Heckman, J.J.: Sample Selection Bias as a Specification Error, in: Econometrica, 47/1 (1979), 153-61; Wooldridge, J.M.: Econometric Analysis of Cross Section and Panel Data, a.a.O., 805-808. 
sind, ${ }^{50}$ schließen wir für die zweite Stufe die quadrierten und kubischen Zeitzählervariablen aus dem Modell aus.

\section{Resultate}

In Tabelle 2 sind die Regressionsresultate von zwei Cragg-Spezifikationen aufgeführt. Die linke Spalte zeigt jeweils das Probit-Selektionsmodell für die Wahrscheinlichkeit, dass die abhängige Variable über dem Schwellenwert von 0 liegt (Selektion, erste Stufe). Die rechte Spalte zeigt jeweils das gestutzte Regressionsmodell für diejenigen Beobachtungen, bei denen der Schwellenwert überschritten wurde (Ergebnis, zweite Stufe). Die wechselnden Vorzeichen zwischen den beiden Stufen sind ein Indiz für die Überlegenheit des Cragg-Modells gegenüber einer Tobit-Spezifikation in unserem Kontext. Nicht zuletzt aufgrund der Ergebnisse unserer deskriptiven Analyse fügen wir das Modellpaar (2) hinzu, um der möglichen Interaktion zwischen Koalitionstyp und parteipolitischer Färbung Rechnung zu tragen.

Tabelle 2: Wahrscheinlichkeit und Umfang fiskalischer Konsolidierungen (Cragg-Modelle)

\begin{tabular}{l|cc|cc}
\hline & \multicolumn{2}{|c|}{$(1)$} & \multicolumn{2}{c}{$(2)$} \\
& Selektion & Ergebnis & Selektion & Ergebnis \\
\hline Koalitionstyp & $-0,43 * * *$ & $1,01 *$ & $-0,71 * * *$ & $0,95 *$ \\
Parteipolitische Zusam- & $(0,12)$ & $(0,53)$ & $(0,14)$ & $(0,54)$ \\
mensetzung & $0,36 *$ & $-1,02 * *$ & 0,11 & $-1,13 * *$ \\
Parteipolitische Zusam- & $(0,19)$ & $(0,49)$ & $(0,29)$ & $(0,45)$ \\
mensetzung & & & $0,69 * *$ & 0,21 \\
$*$ Koalitionstyp & & & $(0,34)$ & $(0,69)$ \\
Fiskalischer Problem- & $0,22 * * *$ & $0,30 * *$ & $0,20 * * *$ & $0,29 * *$ \\
druck & $(0,05)$ & $(0,12)$ & $(0,05)$ & $(0,12)$ \\
Arbeitslosigkeit & 0,05 & 0,02 & 0,06 & 0,02 \\
& $(0,04)$ & $(0,04)$ & $(0,04)$ & $(0,04)$ \\
Wahltermin & $-0,29 * * *$ & 0,35 & $-0,30 * * *$ & 0,35 \\
& $(0,10)$ & $(0,26)$ & $(0,10)$ & $(0,25)$ \\
Stimmendifferenz & $-0,05 * * *$ & $0,08 * *$ & $-0,05 * * *$ & $0,08 * *$ \\
& $(0,02)$ & $(0,04)$ & $(0,02)$ & $(0,04)$ \\
& & &
\end{tabular}




\begin{tabular}{l|cc|cc}
\hline & \multicolumn{2}{|c|}{$(1)$} & \multicolumn{2}{c}{$(2)$} \\
& Selektion & Ergebnis & Selektion & Ergebnis \\
\hline Wahltermin & $0,02 * *$ & $-0,04 * *$ & $0,02 * *$ & $-0,04 * *$ \\
* Stimmendifferenz & $(0,01)$ & $(0,02)$ & $(0,01)$ & $(0,02)$ \\
Institutionelle Beschrän- & $0,07 *$ & $-0,27 * *$ & 0,05 & $-0,28 * *$ \\
kungen & $(0,04)$ & $(0,13)$ & $(0,04)$ & $(0,13)$ \\
Zeitzähler & $-0,76^{* * *}$ & $-0,21$ & $-0,80 * * *$ & $-0,22$ \\
& $(0,14)$ & $(0,35)$ & $(0,14)$ & $(0,35)$ \\
Zeitzähler & $0,08 * * *$ & 0,02 & $0,08 * * *$ & 0,02 \\
& $(0,02)$ & $(0,04)$ & $(0,02)$ & $(0,04)$ \\
Zeitzähler & & $-0,00$ & $-0,00 * * *$ & $-0,00$ \\
Konstante & $-0,00 * * *$ & $(0,00)$ & $(0,00)$ & $(0,00)$ \\
& $(0,00)$ & $-0,38$ & 0,79 & $-0,35$ \\
Sigma & 0,61 & $(1,04)$ & $(0,52)$ & $(1,07)$ \\
& $(0,49)$ & $1,12 * * *$ & & $1,12 * * *$ \\
\hline Pseudo $\mathrm{R}^{2}$ & & $(0,17)$ & & $(0,17)$ \\
Wald chi ${ }^{2}$ & & 0,21 & 0,32 & 0,21 \\
$N$ & & 431,96 & 132,98 & 650,75 \\
\hline & 0,31 & 0,00 & 0,00 & 0,00 \\
& 132,09 & 159 & 527 & 159 \\
\hline
\end{tabular}

Anmerkung: Selektion: Probit-Selektionsmodell für die Wahrscheinlichkeit fiskalischer Konsolidierung; random-effects-Modelle. Ergebnis: gestutzte Regressionsmodelle für den Umfang fiskalischer Konsolidierung. Jeweils panel-robuste Standardfehler in Klammern. $* \mathrm{p}<0,1 ; * * \mathrm{p}<0,05 ; * * * \mathrm{p}<0,01$. Koalitionstyp: 1 für übergrosse Koalitionen oder Minderheitenregierungen; 0 für minimal winning-Koalitionen und Einparteienregierungen. Parteipolitische Zusammensetzung: 1 für von rechten Parteien dominierte Regierungen; 0 für alle übrigen Regierungen.

Auf Basis dieser Modelle berechnen wir für die erste Stufe die vorhergesagten Wahrscheinlichkeiten für eine fiskalische Konsolidierung in den vier von uns unterschiedenen Subgruppen. Analog berechnen wir den vorhergesagten Umfang der Konsolidierung in diesen vier Gruppen für die zweite Stufe. Die Ergebnisse dieser Berechnungen sind in den Tabellen 3 und 4 dargestellt. Beide Tabellen enthalten auch die Differenzen in den vorhergesagten Wahrscheinlichkeiten bzw. Umfängen zwischen den verschiedenen Kombinationen. Wir stützen unsere 
Interpretation der Ergebnisse primär auf dies intuitiv leichter zugänglichen Tabellen 3 und 4.

Tabelle 3: Vorhergesagte Wahrscheinlichkeiten für fiskalische Konsolidierung

\begin{tabular}{l|c|c|c}
\hline & $\begin{array}{c}\text { Breite } \\
\text { Koalition }\end{array}$ & $\begin{array}{c}\text { Minimale } \\
\text { Koalition }\end{array}$ & Differenz \\
\hline Rechte Regierungen & $35,6 \%$ & $36,3 \%$ & $0,7 \%$ (n.s.) \\
& $(4,8 \%)$ & $(6,7 \%)$ & $15,8 \% * * *$ \\
Nicht-rechte Regie- & $17,6 \%$ & $33,4 \%$ & \\
rungen & $(2,5 \%)$ & $(2,2 \%)$ & \\
Differenz & $18,0 \% * * *$ & $2,9 \%$ (n.s.) & \\
\hline
\end{tabular}

Anmerkung: Vorhergesagte Wahrscheinlichkeitswerte basierend auf durchschnittlichen Discrete-Change-Effekten über alle Beobachtungen hinweg. Basierend auf Modell (2). Robuste Standardfehler in Klammern. ${ }^{*} \mathrm{p}<0,1 ; * * \mathrm{p}<0,05 ; * * * \mathrm{p}<0,01$ (nur für Differenz angegeben).

Tabelle 4: Vorhergesagte Werte des Umfangs fiskalischer Konsolidierung

\begin{tabular}{l|c|c|c}
\hline & $\begin{array}{c}\text { Breite } \\
\text { Koalition }\end{array}$ & $\begin{array}{c}\text { Minimale } \\
\text { Koalition }\end{array}$ & Differenz \\
\hline Rechte Regierungen & 1,02 & 0,69 & $0,33 * *$ \\
& $(0,17)$ & $(0,09)$ & $0,52 * *$ \\
Nicht-rechte Regie- & 1,55 & 1,03 & \\
rungen & $(0,26)$ & $(0,09)$ & \\
Differenz & $0.53 * *$ & $0.34 * *$ & \\
\hline
\end{tabular}

Anmerkung: Vorhersagewerte in Prozent des BIP basierend auf durchschnittlichen Discrete-Change-Effekten über alle Beobachtungen hinweg. Basierend auf Modell (1) (wegen nicht signifikantem Interaktionsterm). Robuste Standardfehler in Klammern. * $\mathrm{p}<0,1 ; * * \mathrm{p}<0,05 ; * * * \mathrm{p}<0,01$ (nur für Differenz angegeben).

Auf den ersten Blick scheinen die Resultate unsere erste Hypothese zu stützen, nach welcher die Wahrscheinlichkeit für ein Austeritätsprogramm unter rechten Regierungen höher als unter nicht-rechten ist (erste Stufe). Allerdings ist dieser Effekt der parteipolitischen Färbung nur innerhalb der breiten Koalitionen klar ersichtlich (vgl. Tab. 3, linke Spalte). Unter den minimalen Koalitionen ist der Unterschied deutlich geringer (36,3\% vs. 33,4\%). Über alle Koalitionstypen im Datensatz hinweg erhöht sich die Wahrscheinlichkeit der Initiierung einer Kon- 
solidierung zwar um durchschnittlich 8,9 Prozentpunkte, wenn sie unter einer rechten Regierung stattfindet (nicht in Tabellen berichtet). ${ }^{51}$ Dieser Effekt ist jedoch nur auf Basis von Modell (1) auf dem 90\%-Niveau signifikant. Im methodisch angemesseneren Modell (2) mit dem erklärungskräftigen Interaktionsterm verfehlt er die Signifikanzschwelle knapp. Hypothese 1 findet also nur zum Teil empirische Bestätigung.

Der Umfang von Konsolidierungen fällt unter rechten Regierungen hingegen konsistent (d.h. über alle Koalitionstypen hinweg) kleiner aus (vgl. Tab 4). Dass der Parteieneffekt auf der zweiten Stufe unabhängig vom Koalitionstyp ist, zeigt auch der statistisch insignifikante Interaktionsterm in Modell (2). Über alle Beobachtungen hinweg fällt im Durchschnitt ein Sparpaket um 0,4 Prozentpunkte signifikant kleiner aus (nicht in Tabellen berichtet), wenn es von einer rechten Regierung beschlossen wird. Dies bestätigt Hypothese 2 .

Der Blick auf den Einfluss des Koalitionstyps auf die Konsolidierungswahrscheinlichkeit liefert ein ähnliches Muster wie für den Parteieneffekt, bestätigt hier aber auch die deskriptiven Befunde. In der Gesamtsicht ist Austeritätspolitik unter breiten Koalitionen weniger wahrscheinlich. Im Durchschnitt sinkt die Wahrscheinlichkeit einer Konsolidierung um 11 Prozentpunkte wenn eine breite Koalition regiert. Beschränkt man sich auf die rechten Regierungen, macht der Koalitionstyp jedoch (wie schon in Abbildung 1 angedeutet) keinen Unterschied mehr (siehe auch Spalte „Differenz“, Tab. 3). Auch Hypothese 3 findet somit nur partiell Bestätigung.

Beim Umfang der Konsolidierungen ist das Muster wieder eindeutig: Wenn breite Koalitionen eine Konsolidierung beschlossen haben, fällt diese - unabhängig von der parteipolitischen Färbung - signifikant größer aus als unter minimalen Koalitionen (vgl. Tab. 4). Im Durchschnitt über alle Beobachtungen hinweg, haben die Sparpakete breiter Koalitionen einen um 0,46 Prozentpunkte des BIP signifikant größeren Umfang.

Die Koeffizienten der Kontrollvariablen liegen in den erwarteten Richtungen, wobei wir insbesondere auf den positiven Einfluss des fiskalischen Problemdrucks, sowohl auf die Wahrscheinlichkeit als auch den Umfang einer Konsolidierung, hinweisen.

51 Für die erste Stufe wurden die durchschnittlichen Discrete-Change-Effekte auf Basis von Modell (2) berechnet. Für die zweite Stufe wurde Modell (1) verwendet, da der Interaktionsterm hier keinen signifikanten Einfluss auf den Umfang der Konsolidierung ausübt. 
Unsere alternative Spezifikation von Heckman-Modellen führt zu substantiell identischen Resultaten mit durchweg kleineren Standardfehlern (siehe Anhang). In weiteren Robustheits- und Sensitivitätsanalysen haben wir insbesondere die Paneldatenstruktur der gepoolten Daten sowie die Auswirkungen einflussreicher Beobachtungen auf unsere Ergebnisse berücksichtigt. Unsere substantiellen Befunde ändern sich dadurch nicht. ${ }^{52}$ Schließlich sind alle Ergebnisse robust gegenüber einer getrennten Operationalisierung von breiten Koalitionen, weshalb die Wirkungsmechanismen sowohl für übergroße Koalitionen als auch Minderheitsregierungen gelten. ${ }^{53}$

Wie interpretieren wir unsere Ergebnisse nun in der Gesamtsicht? Für die zweite Stufe zur Frage des Umfangs von Konsolidierungen lassen die Befunde kaum Zweifel aufkommen. Wir finden sowohl einen Parteieneffekt als auch einen Effekt des Koalitionstyps. Diese beiden Einflüsse sind voneinander unabhängig und damit additiv. Substantiell steigt der Umfang einer Konsolidierung also signifikant an, wenn sie von einer nicht-rechten Regierung durchgeführt wird; und er steigt signifikant weiter an, wenn diese Regierung zudem eine breite Reformkoalition hinter sich hat. ${ }^{54}$ Vorausgesetzt, die grundsätzliche Entscheidung zur Austerität wurde getroffen, sind die umfangreichsten Konsolidierungen somit unter nicht-rechten Regierungen in breiten Koalitionen zu erwarten. Die Konsolidierungen von rechten Regierungen in minimalen Koalitionen sollten am geringsten sein. Dazwischen befinden sich die rechten Regierungen in breiten sowie die nicht-rechten in minimalen Koalitionen - zur relativen Größe derer Sparpakete wir keine Aussagen treffen können.

Für die erste Stufe der grundsätzlichen Wahrscheinlichkeit von Austeritätspolitik finden wir offenbar zwei konditionale Effekte: Einerseits scheint der positive Effekt von rechten Regierungen nur unter minimalen Koalitionen Gültigkeit zu haben; anderseits finden wir den positiven Effekt minimaler Koalitionen nur für nicht-rechte Regierungen. Vor diesem Hintergrund halten wir die folgende Inter-

52 Bei der Spezifikation von fixed-effects-Modellen verliert der Parteieneffekt teils auch in der zweiten Stufe an Signifikanz. Allerdings sprechen sowohl methodische (z.B. Hausman-Tests, vgl. Hausman, $J . A$.: Specification Tests in Econometrics, in: Econometrica, 46/6 (1978), 1251-71, geringe Varianz in der Zeitdimension) als auch inhaltliche Gründe gegen die Spezifikation von fixed-effects und für die Robustheit unserer Ergebnisse. Details zu sämtlichen von uns durchgeführten Sensitivitäts- und Robustheitsanalysen sind auf Anfrage erhältlich.

53 Sowohl übergroße Koalitionen als auch Minderheitsregierungen haben eine signifikant geringere Konsolidierungswahrscheinlichkeit (unter nicht-rechten Regierungen), jedoch einen größeren Konsolidierungsumfang (wobei der Unterschied zu den minimalen Koalitionen bei den übergroßen Koalitionen stärker ausfällt als bei den Minderheitsregierungen und bei Letzteren das 90\%-Signifikanzniveau knapp verfehlt).

54 Das gleiche ist auch für die umgekehrte Reihenfolge zu erwarten. 
pretation auf Basis der vorhergesagten Wahrscheinlichkeiten (vgl. Tab. 3) für angemessen: Sowohl rechte Regierungen als auch minimale Koalitionen erhöhen die Wahrscheinlichkeit der Initiierung eines Konsolidierungsprogramms. Die beiden Effekte sind aber nicht additiv: D.h., wenn eines bereits gegeben ist (eine rechte Regierung oder eine minimale Koalition), dann erhöht das jeweils andere die Wahrscheinlichkeit eines Sparpakets nicht signifikant weiter. Somit ist die Wahrscheinlichkeit für ein Sparprogramm sowohl unter rechten Regierungen, unter minimalen Koalitionen als auch unter einer Kombination aus beidem hoch. In allen drei Szenarien ist sie in etwa gleich hoch. Ein Befund lässt jedoch kaum Zweifel zu: Unter nicht-rechten Regierungen in einer breiten Koalition ist eine fiskalische Konsolidierung eher unwahrscheinlich - wenn sie aber stattfindet (siehe oben), ist sie tendenziell sehr umfangreich.

\section{Resultate}

Welche politischen Voraussetzungen müssen gegeben sein, damit Regierungen den Staatshaushalt konsolidieren? Dies war unsere Ausgangsfrage. Wir zeigen einerseits, dass fiskalische Konsolidierungen unter rechten Regierungen und minimalen Koalitionen häufiger auftreten als unter Parteien der politischen Mitte und Linken in einer breiten Reformkoalition. Andererseits finden wir für den Umfang der Konsolidierungsmaßnahmen ein weitgehend entgegengesetztes Muster: Die Sparpakete rechter Regierungen sind kleiner als diejenigen nichtrechter Regierungen; ebenso sind die Sparpakete minimaler Koalitionen kleiner als jene, die von breiten Koalitionen durchgeführt werden.

Unsere Befunde weisen darauf hin, dass bei der Analyse von Austeritätspolitik dem Stufencharakter des politischen Entscheidungsprozesses sowohl konzeptionell als auch methodisch Rechnung getragen werden sollte. Nur so ist es möglich, die aus verschiedenen theoretischen Schulen ableitbaren relativen Einflüsse politischer Variablen empirisch vollständig zu erfassen und somit theoretisch widerspruchsfrei ,unter einen Hut" zu bringen.

So stützen unsere Ergebnisse auf der einen Seite sowohl die traditionelle Vermutung, dass Parteien - ganz im Sinne der klassischen links-rechts-Dichotomie auch in Zeiten der Austerität (noch) einen Unterscheid machen; als auch dass eine hohe Zahl von Vetospielern einer politischen Veränderung grundsätzlich wenig zuträglich sind.

Auf der anderen Seite stützen unsere Ergebnisse aber auch die Vermutung, dass Verhandlungen und Kompromissbildung in modernen Gesellschaften hocheffizi- 
ente Verfahren zur Durchsetzung umfassender Politikveränderungen darstellen, und dass gerade die Parteien der politischen Mitte und Linken Erfolg darin haben, grundlegende Reformen des Wohlfahrtstaates umzusetzen. 


\section{Anhang}

Tabelle 5: Wahrscheinlichkeit und Umfang fiskalischer Konsolidierungen (Heckman-Modelle)

\begin{tabular}{|c|c|c|c|c|}
\hline & \multicolumn{2}{|c|}{ (1) } & \multicolumn{2}{|c|}{ (2) } \\
\hline & Selektion & Ergebnis & Selektion & Ergebnis \\
\hline Koalitionstyp & $\begin{array}{c}-0,45 * * * \\
(0,11)\end{array}$ & $\begin{array}{l}0,62 * * \\
(0,25)\end{array}$ & $\begin{array}{c}-0,72 * * * \\
(0,15)\end{array}$ & $\begin{array}{l}0,77 * * \\
(0,31)\end{array}$ \\
\hline $\begin{array}{l}\text { Parteipolitische Zusam- } \\
\text { mensetzung }\end{array}$ & $\begin{array}{c}0,37 * * \\
(0,17)\end{array}$ & $\begin{array}{c}-0,49 * * * \\
(0,18)\end{array}$ & $\begin{array}{c}0,12 \\
(0,24)\end{array}$ & $\begin{array}{c}-0,36 * * * \\
(0,13)\end{array}$ \\
\hline $\begin{array}{l}\text { Parteipolitische Zusam- } \\
\text { mensetzung } \\
\text { * Koalitionstyp }\end{array}$ & & & $\begin{array}{l}0,68 * * \\
(0,33)\end{array}$ & $\begin{array}{l}-0,37 \\
(0,40)\end{array}$ \\
\hline $\begin{array}{l}\text { Fiskalischer Problem- } \\
\text { druck }\end{array}$ & $\begin{array}{c}0,22 * * * \\
(0,05)\end{array}$ & $\begin{array}{c}0,10 \\
(0,06)\end{array}$ & $\begin{array}{c}0,21 * * * \\
(0,05)\end{array}$ & $\begin{array}{c}0,10 \\
(0,06)\end{array}$ \\
\hline Arbeitslosigkeit & $\begin{array}{c}0,05 * * * \\
(0,02)\end{array}$ & $\begin{array}{l}-0,01 \\
(0,02)\end{array}$ & $\begin{array}{c}0,06 * * * \\
(0,01)\end{array}$ & $\begin{array}{l}-0,01 \\
(0,02)\end{array}$ \\
\hline Wahltermin & $\begin{array}{c}-0,28 * * * \\
(0,10)\end{array}$ & $\begin{array}{l}0,24 * * \\
(0,12)\end{array}$ & $\begin{array}{c}-0,29 * * * \\
(0,10)\end{array}$ & $\begin{array}{l}0,25 * * \\
(0,12)\end{array}$ \\
\hline Stimmendifferenz & $\begin{array}{c}-0,05 * * * \\
(0,02)\end{array}$ & $\begin{array}{c}0,05 * * * \\
(0,02)\end{array}$ & $\begin{array}{c}-0,05 * * * \\
(0,02)\end{array}$ & $\begin{array}{c}0,05 * * * \\
(0,02)\end{array}$ \\
\hline $\begin{array}{l}\text { Wahltermin } \\
\text { * Stimmendifferenz }\end{array}$ & $\begin{array}{l}0,02 * * \\
(0,01)\end{array}$ & $\begin{array}{c}-0,02 * * * \\
(0,01)\end{array}$ & $\begin{array}{l}0,02 * * \\
(0,01)\end{array}$ & $\begin{array}{c}-0,02 * * * \\
(0,01)\end{array}$ \\
\hline $\begin{array}{l}\text { Institutionelle Beschrän- } \\
\text { kungen }\end{array}$ & $\begin{array}{l}0,07 * \\
(0,04)\end{array}$ & $\begin{array}{l}-0,11 * \\
(0,06)\end{array}$ & $\begin{array}{c}0,05 \\
(0,04)\end{array}$ & $\begin{array}{l}-0,10 \\
(0,06)\end{array}$ \\
\hline Zeitzähler & $\begin{array}{c}-0,73 * * * \\
(0,13)\end{array}$ & $\begin{array}{l}0,08 * \\
(0,04)\end{array}$ & $\begin{array}{c}-0,77 * * * \\
(0,13)\end{array}$ & $\begin{array}{c}0,08 * * \\
(0,04)\end{array}$ \\
\hline Zeitzähler ${ }^{2}$ & $\begin{array}{c}0,07 * * * \\
(0,02)\end{array}$ & & $\begin{array}{c}0,07 * * * \\
(0,02)\end{array}$ & \\
\hline Zeitzähler ${ }^{3}$ & $\begin{array}{c}-0,00 * * * \\
(0,00)\end{array}$ & & $\begin{array}{c}-0,00 * * * \\
(0,00)\end{array}$ & \\
\hline Konstante & $\begin{array}{c}0,59 \\
(0,37)\end{array}$ & $\begin{array}{c}0,83 * * \\
(0,35)\end{array}$ & $\begin{array}{c}0,77 * * \\
(0,36)\end{array}$ & $\begin{array}{l}0,74 * \\
(0,40)\end{array}$ \\
\hline Rho & & $\begin{array}{c}-0,46 * * * \\
(0,13)\end{array}$ & & $\begin{array}{c}-0,45 * * * \\
(0,14)\end{array}$ \\
\hline
\end{tabular}




\begin{tabular}{|c|c|c|c|c|}
\hline & \multicolumn{2}{|c|}{ (1) } & \multicolumn{2}{|c|}{ (2) } \\
\hline & Selektion & Ergebnis & Selektion & Ergebnis \\
\hline Wald chi $^{2}$ & \multicolumn{2}{|c|}{71,98} & \multicolumn{2}{|c|}{73,69} \\
\hline $\operatorname{Pr}>\mathrm{chi}^{2}$ & \multicolumn{2}{|c|}{0,00} & \multicolumn{2}{|c|}{0,00} \\
\hline$N$ & \multicolumn{2}{|c|}{$527(159)$} & \multicolumn{2}{|c|}{527 (159) } \\
\hline
\end{tabular}

Anmerkung: Selektions- und Ergebnisgleichung mit panel-robusten Standardfehlern in Klammern. * $\mathrm{p}<0,1 ; * * \mathrm{p}<0,05 ; * * * \mathrm{p}<0,01$. 